\title{
Optical and electrical diagnostics of microdischarges at moderate to high pressure in argon
}

\author{
B.N. Sismanoglu* and C.L.A. Cunha \\ Departamento de Física, Instituto Tecnológico de Aeronáutica, \\ Comando-Geral de Tecnologia Aeroespacial, 12228-900, São José dos Campos, SP, Brasil \\ M.P. Gomes and R. Caetano \\ Laboratório de Óptica e Espectroscopia, Departamento de Física, Instituto Tecnolgico de Aeronáutica, \\ Comando-Geral de Tecnologia Aeroespacial, 12228-900, São José dos Campos, SP, Brasil \\ K.G. Grigorov \\ Institute of Electronics, Bulgarian Academy of Science, 72 Tzarigradsko Chaussee, Sofia 1784, Bulgária
}

(Received on 21 October, 2010)

\begin{abstract}
Microdischarges at moderate to high pressure in argon were investigated. A hole opening diameter of 500 $\mu \mathrm{m}$ direct current (dc) microhollow cathode discharges (MHCD) were characterized by electrical measurements and optical emission spectroscopy (OES) for pressures ranging between 90 and 800 Torr and current from 5 to $20 \mathrm{~mA}$. Current-voltage characteristic curves were obtained as a function of the pressure for this hole diameter. MHCD enables stable dc discharges for molybdenum electrodes material at constant $\mathrm{Ar}+2 \% \mathrm{H}_{2}$ flow of 0.03 $\ell /$ min. Optical emission spectroscopy and analysis of the spectral line broadening of plasma line emissions were performed in order to measure gas discharge parameters. Electron number densities were obtained from $\mathrm{H}_{\beta}$ Balmer line $\left(\sim 10^{14} \mathrm{~cm}^{-3}\right)$. For the above mentioned discharge conditions, gas temperature was estimated to be $550-850 \mathrm{~K}$ from $\mathrm{OH}$ rotational bands. Excitation temperature was measured based on two lines method (from atomic Mo lines) and from $4 p-4 s$ and $5 p-4 s$ Ar radiative transitions. Hydrogen atom temperature was measured for 800 Torr $(\sim 12000 \mathrm{~K})$.
\end{abstract}

Keywords: Microdischarges, microhollow cathode discharges; optical emission spectroscopy.

\section{INTRODUCTION}

Following Schoenbach group studies about microdischarges - notably of microhollow cathode discharges (MHCD) at moderate to atmospheric pressure - the operation and applications of these kinds of microplasmas has attracted the attention of researchers worldwide due to the convenience and easiness of operation, besides the low cost [110]. These non-thermal microplasmas, where the electron temperature is much higher than the gas temperature (here, electron temperature is associated with the mean electron energy), allied to small size and low power consumption, is an attractive to the plasma applications possibilities in industry, like surface treatment, generation of UV and VUV radiation, reduction of pollutants, gas lasers, biological decontamination, thin film deposition, mainly in a high pressure operation $[1,2]$.

These microdischarges consist of a cathode with a borehole and an arbitrarily shaped anode, separated by an insulator, where a hole is drilled through a cathode-mica-anode sandwich structure. Both current-voltage characteristics and Paschen's curves have been studied previously [6-8] for different pressures and hole diameters. They showed three distinct modes of operation: abnormal (at low current), selfpulsed and normal. Stable atmospheric pressure operation is easily obtained in small holes or by employing a certain constant gas flow (dynamic mode). As an example, for a flow of $0.7 \mathrm{l} / \mathrm{min}$ it was possible to ignite a discharge in a $200 \mu \mathrm{m}$ diameter hole using rare gas [11]. For atmospheric pressure operation, the hole diameter should be in the order of $100 \mu \mathrm{m}$

${ }^{*}$ Electronic address: bogoseita.br in a static mode of operation (without gas flowing) [3].

The understanding of physical and chemical processes occurring in this kind of plasma is fundamental for the optimization of the some industrial applications. Reliable MHCD operating at low voltage seems to be useful and important both for industrial and research applications. It is important to notice that at high-pressure, the cathode heating and sputtering is a problem in these devices mainly in static mode of operation, diminishing the lifetime. Therefore, low gas temperature at higher current operation is desirable to avoid these issues. The investigation of plasma parameters, like electron number density $\left(n_{e}\right)$, gas temperature $\left(T_{g}\right)$, electron temperature $\left(T_{e}\right)$ and excitation temperature $\left(T_{\text {exc }}\right)$ generated in MHCD is important to understand the mechanisms that govern these new high-pressure discharges and at the moment, there are no systematic experimental results of plasma parameters $\left(n_{e}, T_{g}, T_{e}\right.$ and $\left.T_{e x c}\right)$ in the literature.

Recent works have shown results on plasma parameters and applications for high pressure $(p)$ quasi-static MHCD, or in a low argon flux, and now they will be presented. For diagnostics and application of an analytical plasma, Miclea et al [12] have measured $n_{e}=9 \times 10^{15} \mathrm{~cm}^{-3}, T_{g}=2000 \mathrm{~K}$, $T_{e}=1.2 \mathrm{eV}$ for discharge current $I_{d}=3 \mathrm{~mA}$ in dc MHCD at atmospheric pressure. Limitation in these applications is the low current and reduced lifetime of the microstructures due to overheating, or sputtering of the micro-hole, at high pressure. Investigating excimer emission from MHCD, Moselhy et al [13] found $n_{e}=1 \times 10^{15} \mathrm{~cm}^{-3}$ in atmospheric pressure, for flowing argon at a rate of $0.04 \ell / \mathrm{min}$ in the gas chamber (gas temperature was not measured). These authors estimated $T_{e}=1.2 \mathrm{eV}$ for dc operation and $2.25 \mathrm{eV}$ in the pulsed mode. Naming micro-structured-electrode arrays, Penache et al [14] have investigated static dc MHCD in pure 
Ar for pressure ranging from 50 to 400 mbar. From spectroscopic measurements of the absorption line profiles they found $T_{g}=1100 \mathrm{~K}$ and $n_{e}=5 \times 10^{15} \mathrm{~cm}^{-3}$ at $p=400 \mathrm{mbar}$. In a device for water treatment, Yamatake et al [15] implemented dc-driven MHCD in argon flow rate $1 \ell / \mathrm{min}$ for 200 $\mu \mathrm{m}$ hole diameter and they have only measured the currentvoltage characteristics, showing abnormal mode of operation. Otherwise, we showed that a dynamic mode MHCD can be operated with intense gas flow in a low gas temperature [11].

In this work optical emission spectroscopy (OES) is used to diagnose the microplasma. In order to measure the basic discharge parameters, an open dc MHCD was used with $\mathrm{Ar} / \mathrm{H}_{2}=98 / 2$ at moderate to high pressure and total flow $0.03 \mathrm{l} / \mathrm{min}$. Plasma parameters were determined from spectral line intensity and broadening. Stark and van der Waals broadenings of the Hydrogen $\mathrm{H}_{\alpha}, \mathrm{H}_{\beta}$, and argon atom $\mathrm{Ar}$ $603.213 \mathrm{~nm}$ and $565.070 \mathrm{~nm}$ lines were used to measure the gas temperature and electron number density of the microplasma for a range of pressure and current. Therefore, we study the variation of the electron number density with pressure and current. In order to analyze the radiation emission from the cathode surface, Mo sputtered and excited atoms could be used to determine $T_{e x c}$ and through OES of $\mathrm{OH}$ (from residual water vapor) the gas temperature could be estimated.

\section{EXPERIMENTAL SET-UP}

The electrodes are made of approximately $100 \mu \mathrm{m}$ thick molybdenum foils separated by a $250 \mu \mathrm{m}$ spacer of mica with $500 \mu \mathrm{m}$ hole diameter. Sustained glow discharge voltages were in the range of $200-400 \mathrm{~V}$ for $\mathrm{Ar} / \mathrm{H}_{2}=98 / 2$ gas mixture at $0.03 \mathrm{l} / \mathrm{min}$ total gas flow. The discharge currents $\mathrm{I}_{d}$ varied between 1 and $20 \mathrm{~mA}$. The operating gas mixture was running from moderate to high-pressure, between 90 and 800 Torr. The electrode system was placed in a cylindrical quartz chamber with $10 \mathrm{~cm}$ diameter and $16 \mathrm{~cm}$ length. Before each experiment the chamber was cleaned, dried and evacuated to about 1 mTorr. Then, the discharge chamber was filled with pure argon gas and placed at the desired pressure. Ballast resistors of $55 \mathrm{~K} \Omega$ were located to the cathode side and were used to limit the discharge current. A picoammeter model Keithley 414S and Minipa digital voltmeter were used to measure the current and voltage in the prebreakdown phase.

Figure 1 shows schematically the MHCD device and the optical apparatus for emission spectroscopy measurements. For spectral investigations the radiation emitted from the cathode borehole was focused onto the optical fiber $1 \mathrm{~mm}$ i.d. aperture by means of convergent lens. This radiation was send onto the entrance slit (width of $100 \mu \mathrm{m}$ ) of $1 \mathrm{~m}$ CzernyTurner (Jobin-Yvon) monochromator (THR1000). The radiation was collimated at the exit slit where a photomultiplier tube (PMT) converts photons into an electric signal. The current generated by the PMT was sent to data acquisition electronics, where the signal was processed by software. The apparatus function was obtained from Ar low-pressure lamp and found to be $0.0463 \mathrm{~nm}$.

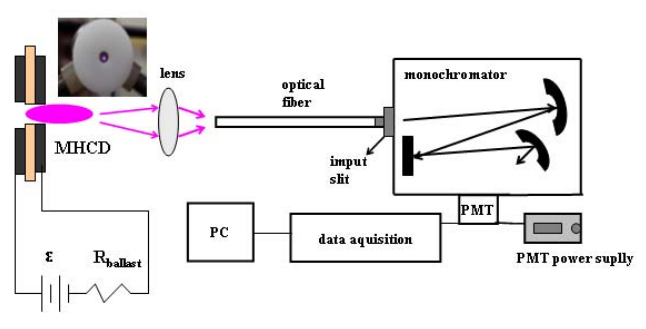

FIG. 1: Experimental set-up showing MHCD and light acquisition; inset: end-on photography of MHCD.

TABLE 1: FWHM for various broadening mechanisms for $\mathrm{H}_{\alpha}$, $\mathrm{H}_{\beta}$, Ar 603.213 and $565.070 \mathrm{~nm}$ lines, at atmospheric pressure $\left(\mathrm{n}_{e}\right.$ in $\mathrm{cm}^{-3}, T$ in $\left.\mathrm{K}\right)$.

\begin{tabular}{ccccc}
\hline Line $(\mathrm{nm})$ & $\Delta \lambda_{\text {Stark }}(\mathrm{nm})$ & $\Delta \lambda_{\text {van Der Waals }}(\mathrm{nm})$ & $\Delta \lambda_{\text {Ressonant }}(\mathrm{nm})$ & $\Delta \lambda_{\text {Doppler }}(\mathrm{nm})$ \\
\hline $\mathrm{H}_{\beta}-486.130$ & GIG-CARD MAPS & $4.528 T_{\mathrm{g}}^{-0.7}$ & $0.513 T_{H_{2}}^{-0.7}$ & $3.48 \times 10^{-4} T_{H}^{0.5}$ \\
$\mathrm{H}_{\alpha}-656.232$ & GIG-CARD MAPS & $5.203 T_{\mathrm{g}}^{-0.7}$ & $0.610 T_{H_{2}^{-0.7}}$ & $4.70 \times 10^{-4} T_{H}^{0.5}$ \\
Ar I-603.213 & See text & $3.931 T_{\mathrm{g}}^{-0.7}$ & 0 & $6.83 \times 10^{-5} T_{\mathrm{g}}^{0.5}$ \\
Ar I-565.070 & See text & $3.310 T_{\mathrm{g}}^{-0.7}$ & 0 & $6.40 \times 10^{-5} T_{\mathrm{g}}^{0.5}$ \\
\hline
\end{tabular}

The main mechanisms responsible for $\mathrm{H}_{\alpha}, \mathrm{H}_{\beta}$, Ar 603.213 and $565.070 \mathrm{~nm}$ lines broadenings were previously investigated [9]. The full-width at half-maximum (FWHM) for these lines are given in Table 1 . In our experimental conditions, both Ar 603.213 and 565.070nm lines are substantially broadened by van der Waals and Stark effects, allowing the estimation of $\mathrm{n}_{e}$ and $\mathrm{T}_{e}$ simultaneously. The gas temperature could be measured both by van der Waals broadening of Ar $603.213 \mathrm{~nm}$ line and $\mathrm{OH}$ radicals $\mathrm{Q}_{1}$ branch. The excitation temperature was determined from the two Mo lines method and from the well-known Boltzmann-plot method using strong Ar $4 p-4 s$ and $5 p-4 s$ transition lines.

In MHCD, where the electron density is about $10^{14} \mathrm{~cm}^{-3}$ at atmospheric pressure, Stark and van der Waals contributions are the most important for these lines broadening, because the line shape is influenced by interactions of the radiating atoms with surrounding particles. Natural and resonance broadening of these lines are usually negligible. The Doppler and instrumental contributions give origin to Gaussian shape line profiles. Stark and van der Waals contributions generate Lorentzian component. The convolution of the Gaussian and Lorentzian components results in a Voigt profile. The GIG-CARD maps [16] for $\mathrm{H}_{\beta}$ line enable to determine the electronic density. In our MHCD, excited non-hydrogenic neutral atoms like Ar lines $603.213 \mathrm{~nm}$ and $565.070 \mathrm{~nm}$ may be used for diagnostic purposes because they are intense and well isolated. Using quasi-static approximation, Griem [17] took into account the small contribution of ion broadening in addition to the electron impact broadening of an isolated line profile. For the line Ar $603.213 \mathrm{~nm}$ the Stark width (FWHM) can be given by:

$$
\begin{aligned}
& \Delta \lambda_{S}(n m)=2\left(0.2206+1.77 \times 10^{-4} T_{e}-1.072 \times\right. \\
& \left.\times 10^{-8} T_{e}^{2}+2.408 \times 10^{-13} T_{e}^{13}\right)\left[1+1.75 \times 10^{-4} n_{e}^{1 / 4} \times\right. \\
& \times\left(0.0628+0.1042 \exp \left(-T_{e} / 4216.168\right) \times\right. \\
& \times\left(1-0.068 n_{e}^{1 / 6} T_{e}^{1 / 2}\right) \times 10^{-17} n_{e}
\end{aligned}
$$


and the Stark width (FWHM) for the Ar $565.070 \mathrm{~nm}$ line is:

$$
\begin{aligned}
& \Delta \lambda_{S}(n m)=2\left(0.2093+1.231 \times 10^{-4} T_{e}-6.045 \times\right. \\
& \times 10^{-9} T_{e}^{2}+1.323 \times 10^{-13} T_{e}^{3}-1.042 \times 10^{-18} T_{e}^{4} \\
& \times\left[1+1.75 \times 10^{-4} n_{e}^{1 / 4} \times(0.00299+0.01513 \times\right. \\
& \left.\times \exp \left(-T_{e} / 1128.45\right)+0.00399 \exp \left(-T_{e} / 14592.93\right)\right) \times \\
& \left.\times\left(1-0.068 n_{e}^{1 / 6} T_{e}^{-1 / 2}\right)\right] \times 10^{-17} n_{e}
\end{aligned}
$$

Solving simultaneously these equations, one can obtain $n_{e}$ and $T_{e}$, presuming that the widths of these lines are previously known. Atmospheric-pressure microplasmas are generally in near-partial-local thermodynamic equilibrium (PLTE) $[17,18]$. The excitation temperature may be determined from Ar emission lines intensities using the wellknown Boltzmann plot method. The two-line method was used for this purpose because the cathode surface sputters Mo excited atoms into the negative glow. The estimation of the excitation and gas temperature are fundamental to obtain the electron number density and other parameters of the plasma.

The rotational temperature $\left(\mathrm{T}_{r o t}\right)$ was measured by using the $O H\left(A^{2} \Sigma^{+}, v=0 \rightarrow X^{2} \Pi, v^{\prime}=0\right)$ emission band at $306.357 \mathrm{~nm}$ [9]. The $\mathrm{OH}$ radical emissions in atmospheric pressure plasma are result of water vapor naturally present as impurity in the gas and can be formed by electron impact dissociation $\left(\mathrm{e}(>4.4 \mathrm{eV})+\mathrm{H}_{2} \mathrm{O} \rightarrow \mathrm{H}+\mathrm{OH}\right)$. In MHCD it was observed emission band between 306 and $312 \mathrm{~nm}$. The $T_{r o t}$ value may be taken from the slope of the plot $\ln \left(I_{0} \lambda / A\right)$ as a function of the upper state energy. Here, $I_{0}$ is the line intensity, $\lambda$ is the transition wavelength and $A$ the transition probability. The standard deviation on $T_{r o t}$ is less than $10 \%$. Finally, from $\mathrm{n}_{e}, \mathrm{~T}_{r o t}$ and $\mathrm{T}_{e}$ one can use GIG-CARD maps for $\mathrm{H}_{\alpha}$ to estimate $H(n=3)$ atom temperature.

\section{RESULTS AND DISCUSSION}

In Fig. 2 is shown the evolution of the current with applied voltage to an open MHCD for pressure ranging from 12 to 800 Torr. Additionally, in Fig. 3 the discharge appearances for different currents and specifically for 40 Torr pressure are presented. As can be seen low current abnormal mode is present, which is characterized by a positive differential resistance, for pressure up to 40 Torr (Fig. 3a). A self-pulsed mode appearing for currents up to $1.5 \mathrm{~mA}$ can be remarked by a voltage drop with current increase, i.e. negative differential resistance, for pressures ranging from 100 to 800 Torr. This region is characterized by high efficiency in producing of ions and metastables species inside the cathode cavity. The confinement of the discharge inside the hole can be seen by the optical appearance of the glow (Fig. 3b). Increasing the discharge current, one can observe a gradual expansion beyond the cavity, covering the cathode surface (Fig. 3c-d). The normal glow discharge mode is seen for currents greater than $1.5 \mathrm{~mA}$, where the voltage is held constant as the current increases.

Three different methods were employed to measure $\mathrm{T}_{e}$ and $\mathrm{T}_{\text {exc }}$ [9]: 1) two Mo lines method ( $\left.\mathrm{T}_{\text {exc }}\right)$; 2) Boltzmann-plot method, for $\operatorname{Ar} 4 \mathrm{p}-4 \mathrm{~s}$ and $5 \mathrm{p}-4 \mathrm{~s}$ transition lines $\left.\left(\mathrm{T}_{\text {exc }}\right) ; 3\right)$

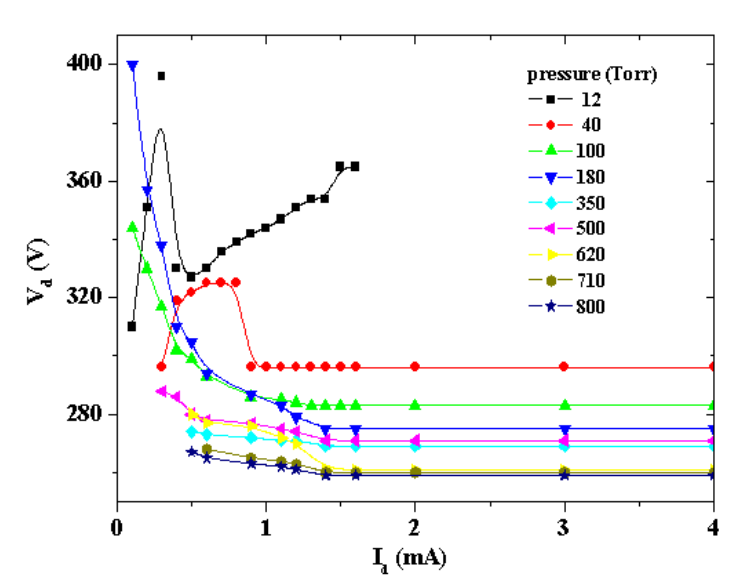

FIG. 2: V-I characteristic curve for a hole diameter of $500 \mu \mathrm{m}$ in $\mathrm{Ar} / \mathrm{H}_{2}=98 / 2$ gas flow.

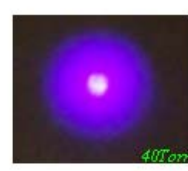

(a)

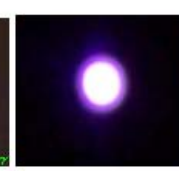

(b)

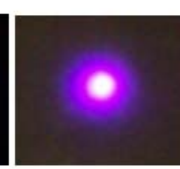

(c)

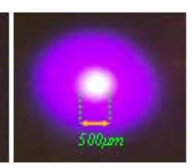

(d)
FIG. 3: End-on photography of the discharge appearance for a hole diameter of $500 \mu \mathrm{m}$ at 40 Torr with the current increasing from 0.3 to $0.7 \mathrm{~mA}$ (a), 0.7 to $1.1 \mathrm{~mA}$ (b), 1.1 to $2.0 \mathrm{~mA}$ (c) and 2.0 to 4.0 $\mathrm{mA}(\mathrm{d})$ (see figure 2).

$\operatorname{Ar} 603.213 \mathrm{~nm}$ and $565.070 \mathrm{~nm}$ lines method, that simultaneously yields $\mathrm{T}_{e}$ and $\mathrm{n}_{e}$ (will be shown later). Based on both, Boltzmann plot and two Mo lines methods, $\mathrm{T}_{\text {exc }} \cong(7000 \pm$ $300) \mathrm{K}$ and $\mathrm{T}_{e} \cong(8500 \pm 1000) \mathrm{K}$ were obtained respectively for current ranging from 5 to $20 \mathrm{~mA}$. For Boltzmannplot the emission intensity of a series of Ar lines from 400 to $820 \mathrm{~nm}$ was recorded and their intensities were corrected for the spectral response of the monochromator. The emission of high intensity $\mathrm{Ar}$ and $\mathrm{Ar}^{+}$lines corresponding to the $5 p-4 s$ and $4 p-4 s(3 d)$ transitions, respectively, indicates high electron temperatures in the investigated region. The difference between $\mathrm{T}_{e}$ and $\mathrm{T}_{e x c}$ was already observed in the literature and the explanation is that the excitation temperature is obtained from the upper excited states distribution, corresponding to a part of electrons of the energy distribution function. $\mathrm{T}_{e}$ obtained with two Ar lines method may be associated with the electrons temperature because this method takes into account a significant part of the electrons energy distribution function. The presence of $\mathrm{Ar}^{+}$lines in the MHCD spectra from 400 to $450 \mathrm{~nm}$ (Fig. 4) shows that electrons with energies between 13 and $24 \mathrm{eV}$ are present in the plasma bulk, which populates the tail of the electrons energy distribution function.

The presence of these argon ions is commonly observed in low-pressure plasmas but not in high-pressure discharges. In regards to two Mo lines method, the region of 440 to 452 $\mathrm{nm}$ was investigated where there are occurrences of atomic lines of excited molybdenum (Fig. 5). These Mo atoms are 
released from the cathode surface by the bombardment of $\mathrm{Ar}^{+}$, and are excited in the region of negative glow. After a Lorentzian fitting of each measured line the intensity was obtained by the area under the fitting curve.

In Fig. 6 the gas temperature was measured using two different methods and it increases with the current increase in good agreement for these methods. The Ar line method for gas temperature measurement procedure is as follow. The $\mathrm{Ar}$ $603.213 \mathrm{~nm}$ line shape is mainly dominated by van der Waals broadening [9]. Doppler broadening is negligible and so the Gaussian component is mainly due to the instrument. After deconvolution, the Lorentzian profile is obtained. From the $\mathrm{H}_{\beta}$ profile $\mathrm{n}_{e}$ may be measured and, finally, $\mathrm{T}_{g}$ may be obtained.

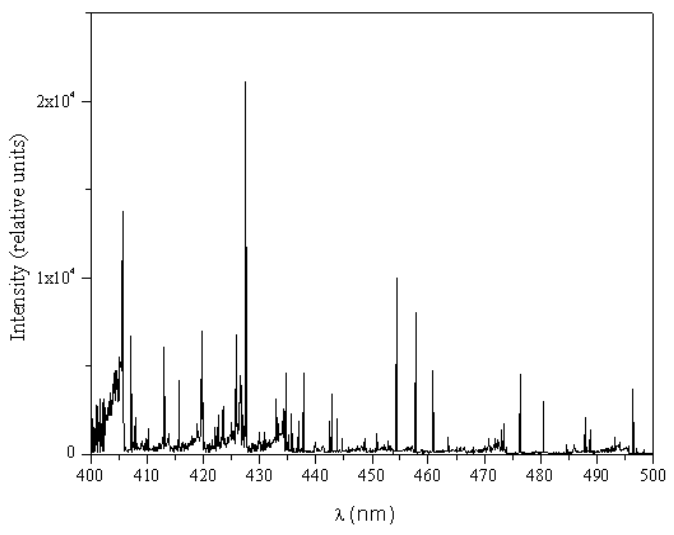

FIG. 4: Spectra of the $A r / H_{2}=98 / 2$ microplasma at $I_{d}=12 m A$.

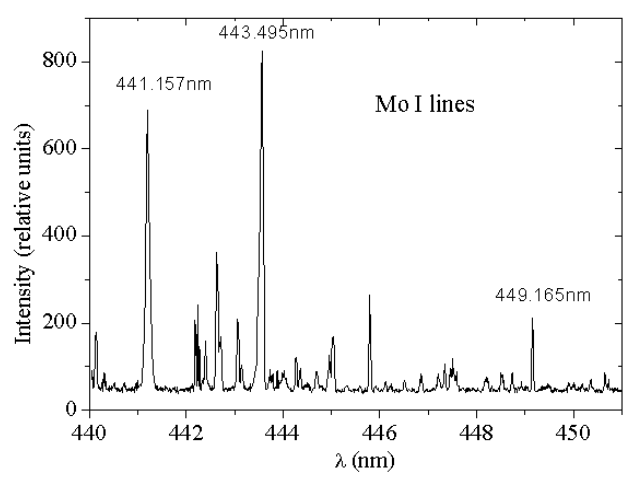

FIG. 5: Mo lines observed in MHCD at atmospheric pressure.

The electron number density $\left(\mathrm{n}_{e}\right)$ was estimated from two methods [9]: a) $\mathrm{H}_{\beta}$ line and b) from $\mathrm{Ar} 603.213 \mathrm{~nm}$ and $565.070 \mathrm{~nm}$ lines methods. From $\mathrm{H}_{\beta}$ line method we can calculate $\mathrm{n}_{e}$ from GIG-CARD maps and it is depicted in Fig. 7 for current ranging from 5 to $20 \mathrm{~mA}$ and pressure from 90 to 800 Torr. From Ar $603.213 \mathrm{~nm}$ and $565.070 \mathrm{~nm}$ lines we obtain $n_{e}$ and $T_{e}$ for 800 Torr by solving simultaneously their Stark FWHM equations and yielded a density of 1.6 to 2.3 $\times 10^{14} \mathrm{~cm}^{-3}$ and $T_{e} \cong(8500 \pm 1000) \mathrm{K}$ for this range of current, showing that $n_{e}$ increases with the current as must be for glow discharges. The thermal nonequilibrium $T_{e}>>T_{g}$ in MHCD shows that this discharge operates in a glow mode

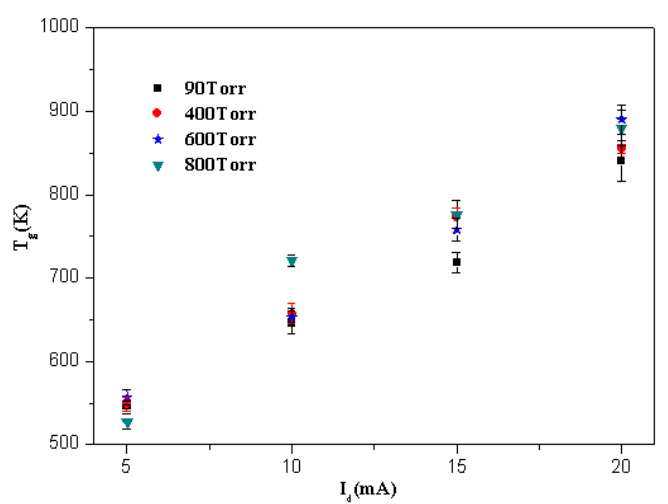

FIG. 6: Gas temperature for MHCD in argon flow for a range of pressure.

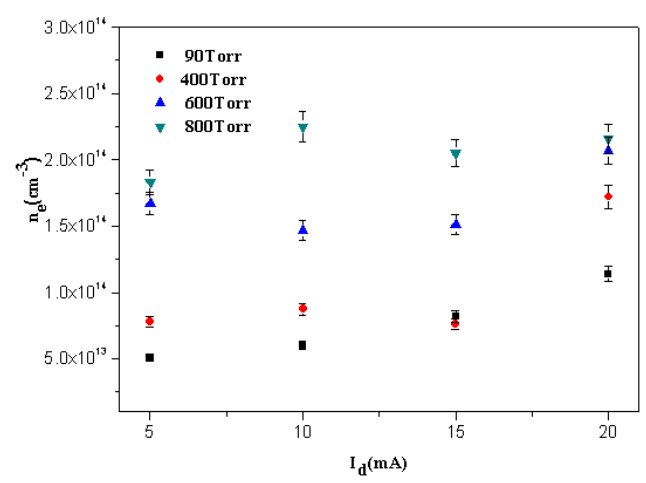

FIG. 7: Electron number density for current ranging from 5 to 20 $\mathrm{mA}$.

and further glow-to-arc transition is possible only at high current. From these results, we obtain hydrogen atom temperature $T_{h} \cong(12000 \pm 1800) \mathrm{K}$ from corresponding $H_{\alpha}$ line (GIG-CARD maps) for this range of current showing that the plasma heavy atoms are not in thermal equilibrium, and $T_{h}>T_{e}>>T_{g}$. The creation process of excited $\mathrm{H}$ atoms may be formed by ion conversion and electron impact dissociation in reactions involving $\mathrm{H}^{+}, \mathrm{H}_{2}^{+}$and $\mathrm{H}_{3}^{+}$in $\mathrm{H}_{2}$ or in $\mathrm{Ar}$ and excited $H$ atoms [9]. For this magnitude of electron density, at 800 Torr, the Debye $d_{D e}$ length is about $3.3 \times 10^{-5} \mathrm{~cm}$. Therefore $\left(2 d_{D e} / D\right)^{2} \sim 10^{-6}(D=$ hole diameter $)$ and this characterize the existence of the plasma and ambipolar diffusion may be used for straightforward estimation of the electron number density $n_{e}\left(I_{d}\right)$ as function of the discharge current [11]. For MHCD hole in a cylindrical cathode configuration, neglecting any loss recombination, we used the continuity equation to deduce $n_{e}\left(I_{d}\right)\left(\mathrm{cm}^{-3}\right)=3.73 \times 10^{17}\left(I_{d} / D_{a}\right)$, where $I_{d}$ is the current in $\mathrm{mA}$ and $D_{a}$ is the ambipolar diffusion coefficient in $\mathrm{cm}^{2} \mathrm{~s}^{-1}$ given by Langevin formula as a function of plasma temperature [11]. For $I_{d}=10 \mathrm{~mA}, T_{g}=$ $730 \mathrm{~K}$, and $D_{a}=8.6 \mathrm{~cm}^{2} \mathrm{~s}^{-1}$ one can obtain $n_{e}=2.0 \times 10^{14}$ $\mathrm{cm}^{-3}$, which is in agreement with our optical results. 


\section{SUMMARY AND CONCLUSIONS}

Emission spectroscopy and power measurements were employed to study dc MHCD in $\mathrm{Ar}+2 \% \mathrm{H}_{2}$ gas flow at moderate to high pressure up to 800 Torr. $\mathrm{H}_{\beta}$ Balmer line and $\mathrm{Ar}$ $603.213 \mathrm{~nm}$ and $565.070 \mathrm{~nm}$ lines were used to characterize the bulk plasma formed in the cathode borehole with diameter $\mathrm{D}=500 \mu \mathrm{m}$. Gas temperature was estimated from $\mathrm{OH}$ radical $Q_{1}$ branch and from $\operatorname{Ar} 603.213 \mathrm{~nm}$ line. The measured gas temperature was in the ranges from 550 to $850 \mathrm{~K}$ for current up to $20 \mathrm{~mA}$, lower than that for static MHCD $(\sim 2000 \mathrm{~K})$. Electron excitation temperature was estimated from both Mo two lines method and Boltzmann plot and was found to be below the electron temperature. The latter was obtained by two Ar lines ( $8500 \mathrm{~K})$. The electron number density was measured both from $\mathrm{H}_{\beta}$ line Stark broadening and from Ar $603.213 \mathrm{~nm}$ and $565.070 \mathrm{~nm}$ lines broadenings increasing with the current. Hydrogen atom $(n=3)$ temperature for 800 Torr was already $12000 \mathrm{~K}$, hotter than the gas and the electron temperature.
[1] K.H. Becker, K.H. Schoenbach and J.G. Eden, J. Phys. D: Appl. Phys. 39, R55-R70 (2006).

[2] J.P. Boeuf, L.C. Pitchford and K.H. Schoenbach, Appl. Phys. Lett. 86, 071501 (2005).

[3] R.H. Stark and K. H. Schoenbach, Appl. Phys. Lett. 74, 25 (1999).

[4] K.H. Schoenbach, M. Moselhy, W. Shu and R. Bentley, J. Vac. Sci. Technol. A 21 (4), 1260 (2003).

[5] K.H. Schoenbach, A El-Habachi, M.M. Moselhy, W .Shi and R.H. Stark, Phys. of Plasma 7, 2186 (2000).

[6] B.N. Sismanoglu and J. Amorim, Eur. Phys. J. Appl. Phys. 41, 165 (2008).

[7] R.S Pessoa, B.N. Sismanoglu, J. Amorim, H. S. Maciel and G. Petraconi, Gas Discharges, Fundamentals and Applications, ed. J. Amorim, Transworld Research Network, Kerala, India, ch. 7, 175, (2007).

[8] M.P. Gomes, B. N. Sismanoglu and J. Amorim, Braz. J. Phys. 39, 25 (2009).

[9] B.N. Sismanoglu, J. Amorim, J.A. Souza-Corra, C. Oliveira and M.P. Gomes, Spect. Acta Part B 64, 1287 (2009).

[10] C. Oliveira, J. A. Souza-Corrâa, M.P. Gomes, B. N. Sisman- oglu and J. Amorim, Appl. Phys. Letters 93, 041503 (2008).

[11] B.N. Sismanoglu, K.G. Grigorov, R. Caetano, M.V.O. Rezende and Y.D. Hoyer, The Eur. Phys. J. D, online first, DOI: 10.1140/epjd/e2010-00219-0 (2010).

[12] M. Miclea, K. Kunze, U. Heitmann, S. Florek, J. Franzke and K. Niemax, J. Phys. D: Appl. Phys. 38, 1709 (2005).

[13] M. Moselhy, K.F. Petzenhauser and K.H. Schoenbach, J. Phys. D: Appl. Phys. 36, 2922 (2003).

[14] C. Penache, M. Miclea, O. Hohn, S. Schoessler, T. Jahnke, K. Niemax and H. Schmidt-Boecking, Plasma Sourc. Sci. and Technol. 11, 476 (2002).

[15] A. Yamatake, J. Fletcher, K. Yasuoka and S. Ishii, IEEE Trans. on Plasma Sci., 341375 (2006).

[16] M. A. Gigosos, M. A. Gonzlez and V. Cardeoso, Spect. Acta Part B 581489 (2003).

[17] H.R. Griem, Plasma Spectroscopy (New York: McGraw-Hill, 1964).

[18] B.N. Sismanoglu, K.G. Grigorov, R.A. Santos, R. Caetano, M.V.O. Rezende, Y.D. Hoyer and V. W. Ribas, The Eur. Phys. J. D, online first, DOI: 10.1140/epjd/e2010-00279-0 (2010). 